\title{
Construire un regard sociohydrologique (2). Le terrain en commun, générateur de convergences scientifiques
}

\author{
Jeanne Riaux ${ }^{1}$, Sylvain Massuel ${ }^{2}$ \\ 1 Anthropologue, IRD, UMR G-EAU (IRSTEA, Ciheam-Iamm, Cirad, Engref, IRD, SupAgro), BP 434, 1004 Tunis, Tunisie \\ 2 Hydrogéologue, IRD, UMR G-EAU (IRSTEA, Ciheam-Iamm, Cirad, Engref, IRD, SupAgro), BP 434, 1004 Tunis, Tunisie
}

Dans un précédent article publié dans NSS, J. Riaux donnait son point de vue d'anthropologue engagée dans la collaboration entre chercheurs de disciplines différentes, l'anthropologie et l'hydrologie (NSS, 21, 1, 2013). Cet article poursuit cette réflexion mais en associant dans l'écriture l'hydrologue. Centrée sur l'analyse conjointe de leur interaction, leur réflexion porte sur le rôle des objets comme passeurs des frontières disciplinaires : les archives (centrales dans le premier article) et le terrain (privilégié dans celui-ci). Dans le projet éditorial de NSS, l'analyse de l'interdisciplinarité comme pratique de recherche est fondamentale. Cette contribution vient s'ajouter à l'ensemble des articles publiés dans la revue et dont l'enjeu est à la fois épistémologique et méthodologique (voir notamment l'article de J. Rouchier et M. Réquier-Desjardins (NSS, 8, 3, 2000, pp. 61-27).

\section{Mots-clés :}

environnement ; ressources naturelles; retour réflexif sur l'interdisciplinarité ; terrain ; Tunisie

Résumé - Second volet d'un travail réflexif sur la construction d'une démarche sociohydrologique, cet article analyse le rôle central du «terrain en commun» dans l'émergence et la structuration de convergences scientifiques entre un hydrogéologue et une anthropologue. Pratique de recherche, support d'observation et de problématisation, le «terrain » est à la fois acteur et produit de l'interdisciplinarité. De cette analyse apparaissent des points communs inattendus entre des démarches disciplinairement éloignées : le rapport au terrain, à l'observation, à l'incertitude et à l'invisibilité des faits considérés. La pratique du terrain en commun aboutit à une vision partagée de l'espace étudié d'où émergent une redéfinition des questions qui font sens et la manière d'y répondre. Cette analyse permet d'expliciter les spécificités d'une recherche interdisciplinaire «fondamentale, inductive et endogène ».

\section{Keywords:}

environment; natural resources; reflexivity on interdisciplinarity; fieldwork; Tunisia

\begin{abstract}
Building a sociohydrological perspective (2). Sharing fieldwork to develop scientific convergences. The paper is the second stage of a reflective work on building a sociohydrological perspective in the wadi Merguellil watershed (Tunisia). A hydrogeologist and an anthropologist relate their common 2-year fieldwork experience and focus on explaining the main stages that led to effective interdisciplinary research. Being together in the field was found to yield the most structuring effects on developing confidence and consistent exchanges between disciplines. Accordingly, sharing common fieldwork is depicted as a key component to build scientific convergences. It is perceived as a research practice and a base for scientific observations and problematization and is both an actor and product of the interdisciplinary approach. Unexpected similarities between distant approaches emerge from the analysis of this experience, like dealing with uncertainty and invisibility of the study objects or the relationship with the field. Practicing fieldwork enables to share an overall view of the study area and in due course generates a new way of asking and answering questions. The reflective work exposes the specific nature of the interdisciplinary research achieved over the shared fieldwork experience. It is considered as "fundamental, inductive and endogenous", because it emerged in a free setting, away from any formal interdisciplinary research program and with no prearranged goal.
\end{abstract}

L'interdisciplinarité ne se décrète pas, elle se construit. Il importe de documenter les voies qu'emprunte cette construction, d'autant plus lorsque qu'il s'agit d'interdisciplinarité « grand-écart» ou «élargie», associant sciences de la nature et sciences de la société (Jollivet, 1992). La construction des collaborations et les interactions qui sous-tendent ce type de recherches représentent en effet un enjeu méthodologique et scientifique.

Auteur correspondant : J. Riaux, Jeanne.riaux@ird.fr 
L'assemblage et l'articulation des apports disciplinaires imposent un dialogue semé de difficultés qu'il faut résoudre collectivement avec entre autres l'élaboration d'un vocabulaire commun, l'explicitation des concepts, la coconstruction d'un objet de recherche ou d'une méthode de travail (cf. p. ex. Barrué-Pastor, 1992 ; Mathieu et al., 1997 ; Jeffrey, 2003). Pour se positionner en démarche scientifique, et non en simple pratique sociale, la progression interdisciplinaire doit être explicitée et justifiée. La réflexivité sur les étapes de la recherche interdisciplinaire est donc une condition de sa rigueur, mais aussi de sa validation (Jollivet et Legay, 2005). Dès lors, la quête prend autant d'importance que les fruits qu'elle produit (Quensière, 1997) et son analyse s'inscrit comme une étape de la pratique interdisciplinaire.

C'est dans cette perspective que s'inscrit la série d'articles intitulée "Construire un regard sociohydrologique» proposée à la revue Natures Sciences Sociétés. Notre ambition est de décrire et d'analyser étape par étape la manière dont se construit une démarche de recherche associant deux disciplines très éloignées: l'anthropologie et l'hydrogéologie. Il s'agit d'abord de partager une expérience interdisciplinaire et les enseignements que l'on en tire, adoptant ainsi la proposition émise par la revue en 1997 en chapeau de l'article de Mathieu et al. : " C'est en s'appuyant sur des expériences de recherche que l'on sortira du terrain spéculatif le débat sur ce que doit être l'interdisciplinarité, et que l'on forgera les savoir-faire indispensables pour la mettre en pratique. » Il s'agit également du choix méthodologique auquel s'adosse notre démarche de recherche : adopter une posture réflexive pour faire régulièrement le point sur le chemin parcouru, les directions prises et celles qui ont été écartées ${ }^{1}$. Un premier article (Riaux, 2013) relate les moments initiaux de ce dialogue interdisciplinaire et la manière dont des documents d'archives se sont constitués en « objets passeurs de frontières » entre des mondes disciplinaires relativement étanches l'un à l'autre. Cet article insiste sur le rôle du «frottement disciplinaire », réalisé à travers ces archives, dans la progression de la réflexion, la construction d'une compréhension et d'une confiance mutuelle permettant l'émergence d'un projet "sociohydrologique ». Le second article présente ici la réflexion sur une étape de cette recherche qui nous associe en tant que jeunes chercheurs, l'un hydrogéologue, l'autre anthropologue, affectés ensemble en Tunisie pour travailler sur un terrain commun. La poursuite de ce dialogue interdisciplinaire révèle un autre objet « passeur de frontières » : le terrain.

1 C'est là une des originalités de la réflexion proposée : dans les recherches interdisciplinaires, lorsque le cheminement est analysé, c'est souvent a posteriori (p. ex. Mathieu et al., 1997 ; Fabre et Cantelaube, 2011) et/ou par un observateur extérieur (p. ex. Barrué-Pastor, 1992 ; Couty, 1993 ; Jeffrey, 2003).
En point de départ à cet exercice autoréflexif, la question de trouver les conditions favorisant la convergence des regards s'est rapidement imposée. Comment deux sciences relevant de deux extrêmes de l'existant (le soussol et les humains) peuvent-elles se rapprocher? Comment trouver les fondements permettant de construire un regard partagé sur une situation et des questions de recherche communes (que l'on appellera sociohydrologiques) qui s'inscrivent autant dans les démarches et interrogations de chaque discipline ? À la suite de Mathieu et al. (1997), nous constatons que l'expérience et la pratique communes du terrain figurent parmi les activités les plus structurantes pour la construction de l'interdisciplinarité, formant le creuset d'une certaine alchimie sociohydrologique. C'est pourquoi nous proposons ici d'explorer la manière dont la pratique commune du terrain permet de générer des convergences scientifiques productrices d'originalités et peut-être d'un renouvellement des modalités d'analyse d'un espace sociohydrologique.

\section{Aux origines du terrain partagé}

Le cadre de notre réflexion est le bassin de l'oued Merguellil en Tunisie centrale (Fig. 1), où de nombreuses recherches se sont succédé, apportant des réponses mais aussi de nouvelles questions sur certains processus hydrologiques complexes, notamment ceux faisant intervenir l'influence des actions humaines sur les régimes hydrologiques (Leduc et al., 2007). Pour faire face à ces interrogations, des chercheurs de l'IRD et de l'Inat (Institut national agronomique de Tunis) ont souhaité adopter une perspective multidisciplinaire, en associant l'économie et l'agronomie à l'hydrologie (Le Goulven et al., 2009). L'ambition pluridisciplinaire a été amplement réalisée, bien que la dimension sociale et politique de la gestion des eaux du Merguellil reste encore à documenter. Notre affectation en Tunisie s'inscrit dans la continuité de ces recherches, avec l'objectif d'aller plus loin dans la compréhension de cet espace.

À travers le premier bilan des travaux effectués par nos prédécesseurs, nous nous accordons sur le fait que des approches pourtant pluridisciplinaires, mais abordant d'un côté la ressource et de l'autre l'usage, ne peuvent produire une vision suffisamment éclairante de ce bassin. Le décloisonnement disciplinaire est nécessaire, en particulier pour dépasser cette séparation ressource/ usage qui s'avère peu opérante. Cet objectif est au centre du programme Dyshyme, « Dynamiques sociohydrologiques en Méditerranée ", que nous coordonnons ensemble et dans lequel cette recherche interdisciplinaire est déployée ${ }^{2}$. Au départ, la notion de regard ou de dynamiques «sociohydrologiques » était

\footnotetext{
2 Projet financé par l'action Sicmed du programme Mistral depuis 2011.
} 


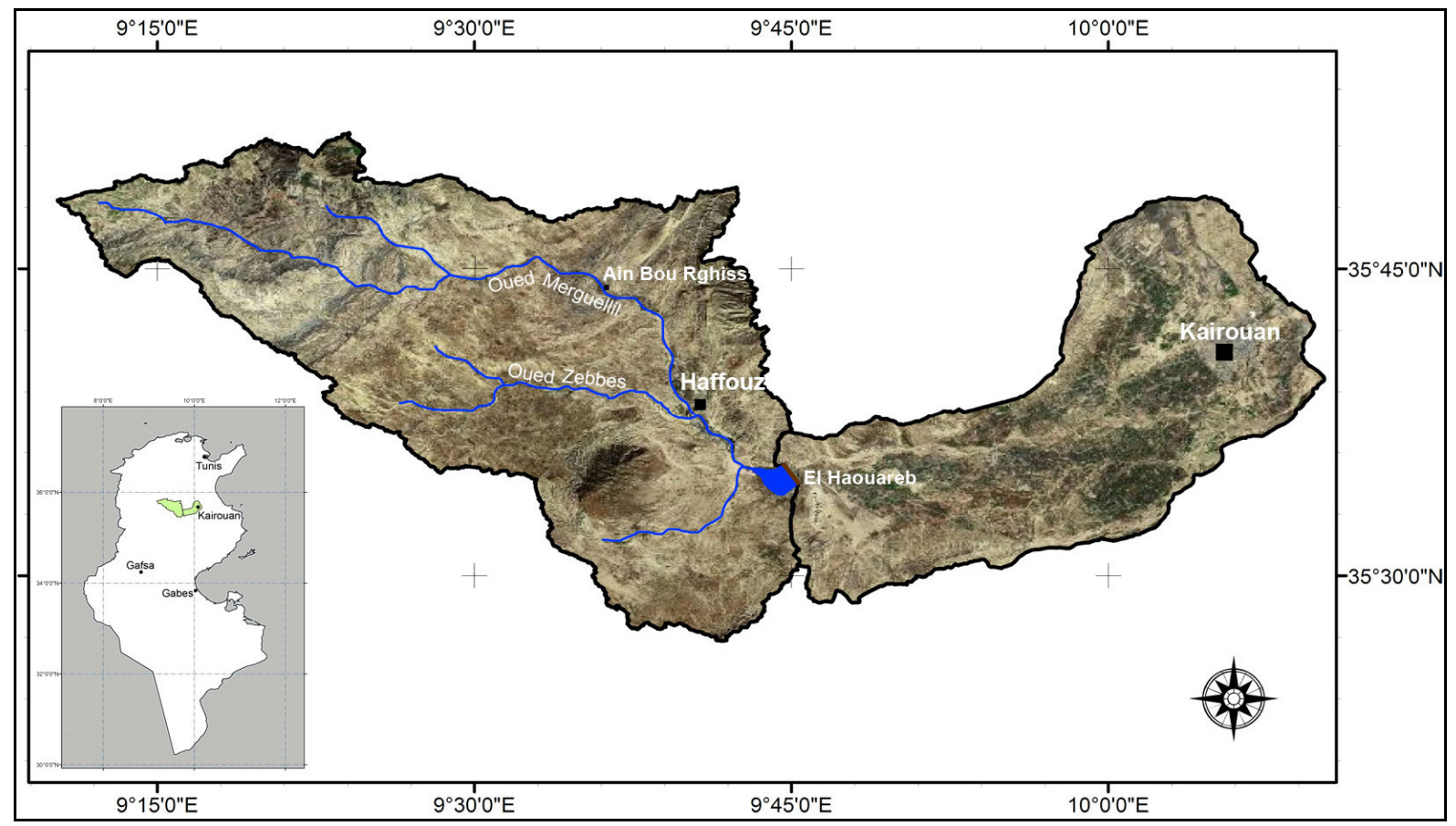

Fig. 1. Le bassin de l'oued Merguellil, en Tunisie centrale.

plus intuitive que réellement construite. Il s'agissait surtout de marquer un changement de regard en associant du « socio » à de «l'hydro ». Mais cet usage du terme "sociohydrologique» ne suffit pas à lui donner un contenu. C'est principalement dans l'interaction entre personnes et avec le terrain que ce projet se construit et acquiert du sens.

\section{Le rapport au terrain, révélateur et catalyseur de convergences}

\section{Un processus d'appropriation partagée du terrain d'étude}

Nos premières interactions scientifiques se sont véritablement déroulées sur l'espace de travail commun au cours de « missions de terrain ». Il s'est d'abord agi pour l'anthropologue de prendre connaissance du terrain des hydrologues, d'observer leurs pratiques de recherche et d'essayer de comprendre la manière dont ils envisagent cet espace, le questionnent et le documentent. L'hydrogéologue a, quant à lui, commencé par donner des clés élémentaires de compréhension à l'anthropologue pour lui permettre de se situer par rapport à ses activités et à sa vision de l'espace hydrologique et des repères qui y font sens. En retour, une autre perception de l'espace s'est ouverte. D'observatrice distante, au fur et à mesure de son appropriation du terrain, l'anthropologue est devenue à son tour initiatrice par ses propres questionnements et centres d'intérêt. Outre l'observation des modes opératoires de nos deux disciplines, ces missions sur le terrain ont permis d'engager des discussions sur la manière dont se pratiquent l'hydrogéologie et l'anthropologie. Nous nous sommes en particulier interrogés sur les méthodes d'observation, la manière de produire des questions de recherche et des données susceptibles d'y apporter des éléments de réponse.

De ces discussions ressort l'importance que tient le terrain dans nos démarches respectives d'un point de vue méthodologique et scientifique, mais aussi d'un point de vue plus personnel. Comme l'exprime l'hydrogéologue, « le terrain n'est pas un lieu anonyme, tout ce qui le concerne nous interpelle, nous intéresse, une liaison intime se crée nécessairement ». Pour l'anthropologue, « le terrain est un lieu d'attraction, un lieu obsédant que l'on veut à tout prix comprendre, embrasser dans son ensemble ». Pour les deux disciplines, il y a tout un processus de transformation d'un espace anonyme en un « terrain » approprié, balisé et reconnu. L'importance des modalités de définition de ce terrain est ici centrale. Chacun d'entre nous construit ses points de repère, identifie des éléments significatifs par rapport à ses objets de recherche. Nous nous approprions donc cet espace à travers un prisme disciplinaire, ce qui conduit chacun de nous à penser qu'il en existe plusieurs formes distinctes, une sorte de segmentation qui rendrait difficile d'envisager le partage. Or le fait même de se rendre ensemble sur le terrain nous fait prendre conscience que cette appropriation peut être commune et qu'il n'y a pas de véritable cloisonnement. Par exemple, l'anthropologue découvre la complexité des « relations surface-souterrain » autour d'un oued, ce qui la conduit à envisager autrement des relations entre puits qui lui semblaient aller de soi. L'hydrogéologue réalise à quel point les relations de 
pouvoir prédominent dans les décisions d'implantation ou de gestion des ouvrages de captage mineurs (puits, canaux) dont la logique hydraulique et/ou économique n'est pas évidente. Chacun révèle simplement à l'autre une facette de ce terrain qui lui était inaccessible ou cachée. Ce qui pourrait être perçu comme une intrusion favorise en fait largement, et de manière continue, la convergence des regards.

\section{Importance de l'observation et centralité du terrain}

De ce processus de partage émerge un élément commun central qui est notre rapport au terrain et, en particulier, la place tenue dans nos démarches scientifiques par l'observation et par la triangulation à partir de sources d'information variées.

Il est bien connu en sciences sociales que l'analyse des faits sociaux ne peut être effectuée par observation directe compte tenu de la complexité des éléments qui les composent et de l'importance qu'y tiennent les « relations ", invisibles par essence. Il est nécessaire de trouver des indices matériels qui permettent de traduire ces faits en éléments observables. En s'intéressant aux eaux souterraines, l'hydrogéologue se heurte lui aussi, de manière très directe à l'invisibilité de son objet d'étude. Il faut là encore trouver des objets à observer qui peuvent témoigner d'une réalité impalpable. Un point commun de nos sciences est donc la nécessité de trouver des moyens de «voir » ce qui ne se voit pas.

Dès lors, nous avons en commun un rapport spécifique à la matérialité du terrain où la médiation des objets joue un rôle central. Pour l'hydrogéologue, ces objets apparaissent de manière plus ou moins évidente: un puits, une source sont des «fenêtres ouvertes » sur les eaux souterraines. Ce sont des lieux privilégiés pour documenter l'invisible. Sinon, les observations sont toujours indirectes, soit par l'utilisation de traceurs chimiques ou de techniques d'imagerie non invasives, soit par la création invasive de ces objets (piézomètres, carottage). Pour l'anthropologue s'intéressant aux rapports eaux/sociétés, fréquenter ces lieux-objets accompagnée d'hydrogéologues permet de découvrir une autre dimension de la richesse qu'ils recèlent en termes de matériau ethnographique. Ainsi, discuter d'un puits avec son propriétaire permet d'identifier la pertinence et l'originalité des savoirs hydrologiques qu'il détient. Cette médiation des personnes qui utilisent, entretiennent, construisent ces objets est aussi bénéfique pour l'hydrogéologue. Elle constitue une source d'enseignements utiles pour la mise en perspective des mesures physiques. Les précieux indices que nous confient nos interlocuteurs peuvent parfois même induire de nouvelles perspectives de recherche.

Cela nous renvoie à un autre point commun de nos disciplines : la production ou l'acquisition de données et les différentes incertitudes associées (représentativité, erreurs, incompréhensions, etc.). Là encore, plusieurs constats permettent de relier nos pratiques de terrain, même si le constat de similitudes n'annule - bien sûr pas ces différences.

\section{Observations et croisement des informations}

Dans sa démarche scientifique, l'hydrogéologue revient constamment au terrain qui est le lieu central de ses activités d'observation et d'expérimentation. Il en est de même pour l'anthropologue. Nos deux disciplines mettent l'observation au cœur de leurs démarches, même si l'on ne regarde pas spontanément la même chose. Nous avons besoin de chercher sur le terrain des éléments qui nous permettent de construire nos hypothèses et de les documenter.

Cette importance accordée au terrain et à l'observation témoigne de la centralité pour nos disciplines, de la production de données les plus fiables possible sur ce que nous cherchons à observer. Or le fait que nos objets d'étude ne soient pas directement observables nous invite à croiser le plus d'informations possible dans l'objectif de pallier le caractère intrinsèquement incertain ou subjectif des données issues de l'observation. Comme l'illustrent certains travaux de l'hydrogéologue (Massuel et al., 2008), le croisement de différentes méthodes d'observation permet de limiter ces contingences. Des protocoles scientifiques doivent être respectés pour réduire les marges d'incertitude de la donnée, mais aussi pour renseigner les conditions de la mesure afin d'en étayer la critique et de lui donner le crédit qu'elle mérite selon son usage (Favreau et al., 2000). De son côté, l'anthropologue ne se cantonne pas à recueillir des paroles d'acteurs qui confirmeraient ou non une hypothèse ; les méthodes de l'ethnographie et de l'entretien qualitatif permettent de croiser les informations pour étayer ou invalider des hypothèses, bref, pour éviter le biais de la surinterprétation (Olivier de Sardan, 1996).

Ce dernier point nous conduit à réfléchir aux spécificités de nos objets de recherche par rapport à nos disciplines respectives. Ces spécificités ont impliqué une ouverture à l'interdisciplinarité dans nos trajectoires de recherches passées et impriment une marque particulière à la démarche que nous mettons aujourd'hui en œuvre.

\section{La pratique commune du terrain : une posture favorable au croisement des regards}

\section{Révélations et postures des disciplines}

L'adoption d'une «réflexivité en miroir » nous a conduits à comparer nos parcours respectifs et en 
particulier la manière dont la recherche sur l'eau pour l'une et sur les milieux semi-arides pour l'autre nous conduisait déjà au-delà des frontières de nos disciplines.

Il y a d'abord l'histoire de nos disciplines qui implique des hybridations. L'hydrogéologie est à la croisée des chemins d'un bon nombre de disciplines scientifiques, parmi lesquelles l'hydrologie et la géologie sont les plus évidentes. L'hydrogéologie ne mobilise donc pas un unique point de vue sur la réalité et paraît de fait plus encline à de nouveaux apports. D'une manière très différente, l'anthropologie centrée sur l'eau implique la prise en compte de champs d'observation relativement marginaux par rapport aux objets d'étude classiques de la discipline. L'observation de systèmes irrigués nécessite de documenter les pratiques hydroagricoles, donc de comprendre le fonctionnement d'un réseau hydraulique et les savoirs associés à l'eau mobilisée (Marzouk, 1989; Aubriot et Riaux, 2013). Cette particularité de l'objet de recherche infléchit donc la démarche disciplinaire en l'ouvrant nécessairement aux apports d'autres spécialités.

Par ailleurs, nos recherches antérieures nous ont amenés à reconnaître l'importance de ne pas regarder seulement la « ressource » pour l'un, ou la «société » pour l'autre, notamment dans des contextes de recherche pour le développement. Pour l'hydrogéologue, le fait d'être confronté à son objet d'étude dans le cadre d'applications au développement a des implications. En milieu semiaride, la donnée hydro(géo)logique est rare et incertaine. Le réflexe scientifique est alors, au cours de l'investigation, de chercher le moindre indice pour qualifier la mesure, toujours dans une perspective d'estimation du niveau de validité à attribuer à une donnée. L'expérience de ses premiers travaux au Niger (Massuel et al., 2011) lui a permis d'entrevoir au travers des échanges avec les acteurs de terrain, la richesse des informations potentiellement disponibles, bien que difficilement accessibles par ses propres outils. Le témoignage des anciens, expliquant que l'évolution de la déforestation au profit des espaces cultivés avait eu lieu depuis les villages, des vallées vers les collines, aurait mérité de plus amples investigations. Pour l'anthropologue, l'un des objectifs de ses recherches sur l'eau en Méditerranée (Riaux, 2006) impliquait de comprendre et d'interpréter les relations sociohydrauliques sur un territoire. L'ignorance des caractéristiques hydrologiques des espaces étudiés a vite constitué un frein à cette recherche : le refus par les irrigants de " voir » une relation entre le tarissement de sources à l'aval et le creusement de puits à l'amont était-il fondé sur les caractéristiques du milieu ou sur des hiérarchies d'accès à l'eau socialement acceptées ? Cette question n'a jamais trouvé de réponse faute de compétences dans le domaine.

Ces éléments concernant notre passé scientifique et la manière dont nous avons essayé de résoudre le problème de l'accès à l'information et de l'incertitude confirment que nous étions déjà, avant de nous rencontrer en Tunisie, dans une posture favorable à la pratique interdisciplinaire. Notre positionnement de départ se caractérise par un mélange de curiosité, d'intérêt mutuel et de nécessité.

\section{Lever les incompréhensions}

Lors des missions sur le terrain, nous discutons de nos démarches, exposons nos méthodes. Il en résulte des réflexions sur nos points communs, comme évoqué précédemment, mais aussi sur nos divergences. Dans certains cas, nos vocabulaires très spécifiques entravent la compréhension. C'est le cas avec les mots «source » ou «tribu » qui se constituent en notions complexes pour une discipline, alors que pour l'autre ils relèvent du sens commun. Des mots comme « usage » peuvent aussi désigner des faits très différents dans les deux disciplines, ou $\mathrm{d}^{\prime}$ autres comme « ressource » sont de véritables concepts dans une discipline et pas dans l'autre, ce qui entraîne des confusions et produit des interprétations erronées. Mais le terrain autorisant les longs échanges, peu de sujets restent en suspens.

Au fil des discussions, nous réalisons que nous avons sur la discipline de l'autre un certain nombre de préjugés que la discussion, parfois animée, permet d'identifier et de déconstruire. Pour l'hydrogéologue, par exemple, la démarche des " sciences sociales » est trop subjective, car dans chaque étude, seules quelques causes des faits sociaux sont identifiées. Pour lui, le type de données produites et le raisonnement qui les accompagne sont par nature biaisés, trop éloignés d'une véritable démarche « scientifique » pour avoir une valeur intrinsèque. De son côté, l'anthropologue s'aperçoit qu'elle a parfaitement intériorisé l'a priori selon lequel les « sciences dures » ont l'impression de détenir des vérités absolues et de laisser peu de place au doute. Ce préjugé est accentué par le fait que ces sciences donnent surtout à voir des résultats objectivés souvent sous une forme totalement opaque pour le profane.

Avec le terrain en commun, nous apprenons à découvrir la discipline de l'autre à travers la démarche sur laquelle elle repose, sa posture scientifique. La dimension matérielle et pratique de l'activité de terrain permet aussi d'illustrer des points difficiles à expliquer plus théoriquement. Par ailleurs, cet exercice d'initiation place le terrain en commun en "épreuve de vérité ». En effet, alors qu'une communication scientifique est par essence un exercice de présentation synthétique et magnifié des travaux réalisés (passant donc sous silence les bricolages et ajustements de la méthode rendus nécessaires, $c f$. p. ex. Latour, 1993), la pratique commune du terrain expose les dessous des modes opératoires. Elle ne permet pas la dissimulation des approximations, des incertitudes et des 
difficultés rencontrées. Cela suscite des échanges marqués par la transparence, essentielle au fondement du rapport de confiance. C'est le dévoilement de cette arrière-cuisine de la science qui a été formateur pour nous deux ; il était nécessaire d'expliciter les pratiques dans leur ensemble, afin que le jugement de l'autre ne soit pas rédhibitoire. Il fallait resituer à la fois ce que l'on « dit », ce que l'on « fait » et ce que l'on « dit qu'on fait», lui donner du sens pour ne pas l'invalider aux yeux de l'autre, instaurer une sorte d'estime mutuelle à l'égard de nos travaux respectifs. Il est toujours difficile de prendre du recul sur ses propres pratiques pour les expliquer, les justifier. Mais sur le terrain, nous percevons mieux la complexité de la démarche scientifique dans laquelle s'insèrent les pratiques de recueil de données de chacun, la manière dont nous mettons en doute et gérons les « incertitudes » inhérentes à nos pratiques de " production de données ». Cet exercice suscite également de la part de « l'autre scientifique » des interrogations spontanées parfois inattendues par leur simplicité ou leur innocence. C'est de fait un révélateur des incompréhensions causées par des regards spécialisés omettant d'exprimer les évidences.

\section{Changements de postures : ouverture et évolution des regards}

Bien sûr, ces mises au point ne suffisent pas à expliquer le fond de la démarche scientifique de chacun. Elles nous ont néanmoins permis d'adopter une posture plus attentive et plus réceptive à la démarche et aux pratiques de l'autre. Nous identifions le fait que nous portons des regards - simplement - différents sur le monde, mais que l'un n'a pas plus de validité que l'autre. Un changement de posture est nécessaire pour s'approprier la manière dont l'autre se représente et conceptualise une situation. Ainsi, comment un anthropologue pourrait-il accepter que l'on réduise les rapports eaux-sociétés à de simples usages, et les usages aux seuls prélèvements quantifiables? En cherchant à comprendre en quoi ces " usages » ont une importance cruciale dans un bilan hydrologique, on comprend qu'il ne s'agit pas de réduire les activités humaines à un chiffre, mais bien d'évaluer des prélèvements dans un stock d'eau. De même, il n'est pas évident pour un hydrogéologue de concevoir que la baisse des niveaux des nappes ne soit pas l'élément capital associé à la notion de surexploitation. Le déséquilibre entre les entrées et les sorties des systèmes aquifères dues aux prélèvements humains n'est effectivement qu'une dimension du phénomène que l'anthropologue ne cherche pas mécaniquement à déconstruire mais bien à penser dans sa globalité.

La levée de ces préjugés donne du crédit aux activités de l'autre. Nous sommes en position d'apprendre l'un de l'autre et d'accepter de ne pas saisir l'ensemble de la démarche disciplinaire sans pour autant émettre un jugement à l'aune de nos références respectives ${ }^{3}$. Ce changement de posture aiguise automatiquement notre intérêt pour les pratiques et pour les apports de l'autre discipline désormais légitimée. L'autre discipline constitue un potentiel à valoriser, à la fois pour les apports qu'elle peut représenter vis-à-vis de chaque démarche, et pour ce que l'association des deux disciplines peut produire. Ce potentiel s'exprime autant d'un point de vue méthodologique que pour la production d'un savoir interdisciplinaire.

\section{La définition commune du terrain : convergence des regards et émergence d'un projet « sociohydrologique »}

Au cours du travail de terrain, nous construisons à la fois un espace intelligible pour nous deux et des questions de recherche partagées. Un schéma évolutif du bassin du Merguellil et des éléments qui le composent nous permet de représenter ce terrain devenu commun (Fig. 2).

\section{Des « nœuds sociohydrologiques » du territoire et de l'analyse}

Plusieurs objets nous permettent de construire l'ossature de cette représentation commune du terrain. Ce sont des éléments qui structurent l'espace, des «lieux » sur lesquels nous nous rendons ensemble avec un regard interrogateur.

Le premier d'entre eux est le barrage El Haouareb. C'est un élément clé de l'espace hydrographique puisqu'il bloque les écoulements de l'oued, représentant ainsi un point de rupture entre un amont "producteur d'eau » et un aval «consommateur» où se développe une agriculture intensive à partir des eaux souterraines. La construction du barrage sur un massif calcaire fissuré a causé la formation d'une émergence. C'est l'une de ces «fenêtres ouvertes » sur les eaux souterraines pour les hydrogéologues : à partir d'analyses chimiques et hydrodynamiques, ce lieu permet de documenter à la fois les relations entre eaux de surface et eaux souterraines et les relations entre plusieurs nappes. C'est aussi un lieu signifiant du point de vue des rapports que les sociétés entretiennent avec l'eau et avec l'espace. Ce barrage représente à la fois un élément marquant de l'histoire et de l'organisation spatiale de la région. Il s'agit en effet d'un lieu de transition entre la plaine de Kairouan et les zones

\footnotetext{
3 Ce point est pourtant considéré par Bruce et al. (2004) comme l'un des principaux éléments de blocage rencontrés dans la mise en œuvre de l'interdisciplinarité lors du $5^{\mathrm{e}}$ Programme-cadre européen.
} 


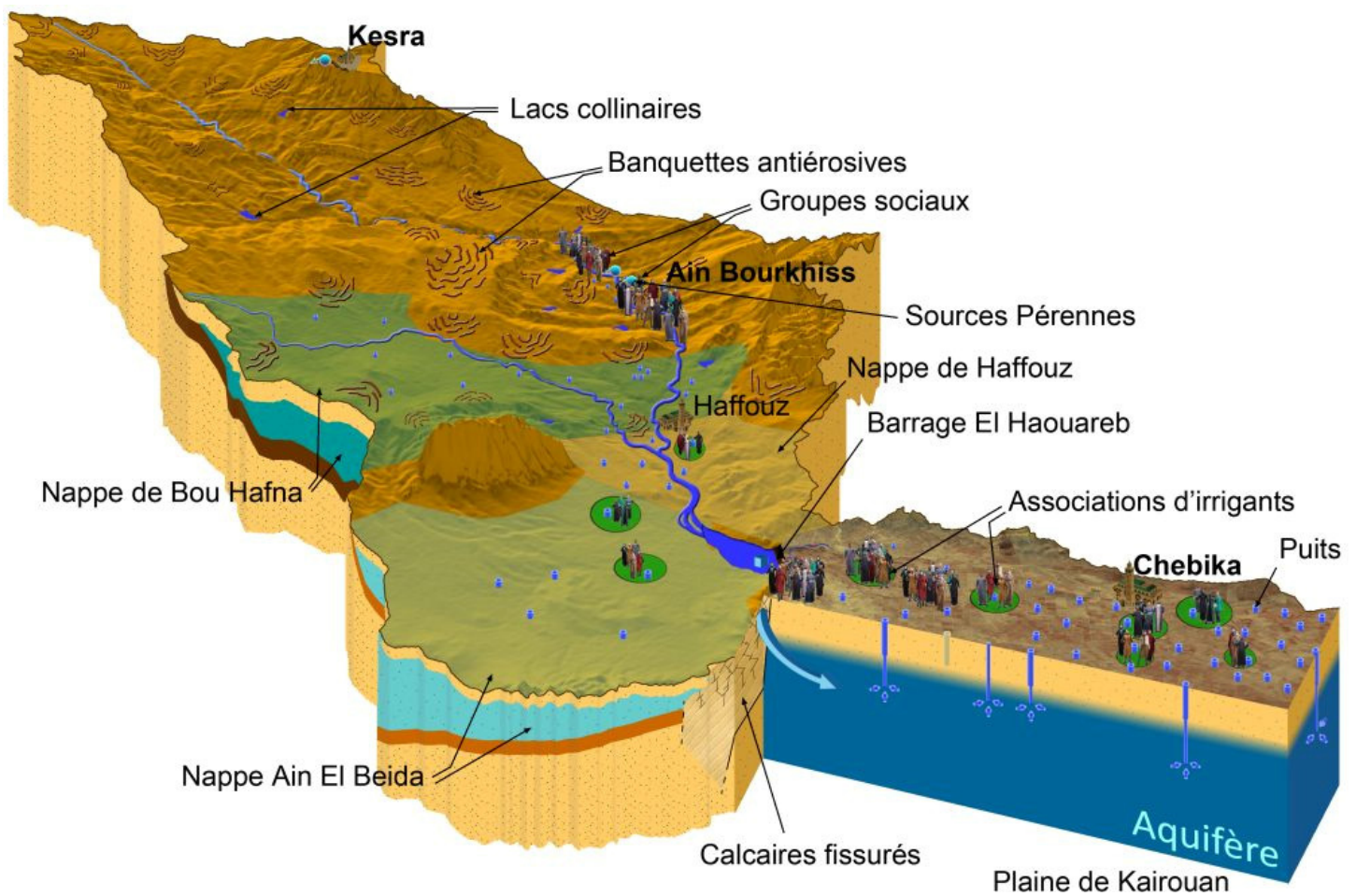

Fig. 2. Schéma « sociohydrologique » actuel du bassin du Merguellil.

de montagne, deux espaces historiquement différenciés. Le second élément important est la plaine de Kairouan et l'aquifère sur lequel elle se trouve. D'un point de vue hydrogéologique, c'est un espace cohérent: l'espacenappe dont l'évolution des réserves témoigne des bouleversements en surface. D'un point de vue anthropologique, la nappe est un objet à part entière, à la fois en tant que « ressource » hydroagricole et en tant qu'élément autour duquel se déploient discours et actions publics. Le troisième élément, une section pérenne de l'oued Merguellil alimentée par plusieurs sources, se situe à l'amont du barrage. Véritable singularité dans le paysage semi-aride pour l'hydrogéologue, ce point de contact naturel entre les eaux souterraines et la surface représente, pour l'anthropologue, un espace socialement cohérent révélateur des trajectoires d'accès à l'eau des groupes sociaux en présence. C'est un lieu qui nous amène à regarder ensemble les mêmes objets : des eaux qui sourdent, des pratiques d'irrigation, des techniques d'exhaure (Fig. 3).

De manière plus fine, au sein de ces différents espaces, des objets retiennent plus précisément notre attention. Les puits, forages, piézomètres, pompages, vestiges de canaux, ouvrages de prise dans l'oued, sources sont autant d'objets sociotechniques ou hydrogéologiques qui représentent des « nœuds sociohydrologiques » du territoire. Ces objets-lieux importants en tant que sources d'informations à la fois pour l'anthropologue et pour l'hydrogéologue sont aussi des objets sur lesquels nos regards se rencontrent, où nos questionnements respectifs s'articulent, se complètent et s'enrichissent. Parfois les réponses trouvées par l'un permettent à l'autre d'approfondir le champ de ses questionnements. Par exemple l'histoire d'un puits permet de relier la profondeur de l'ouvrage avec les niveaux anciens de la nappe et témoigne par conséquent d'évolutions sur le long terme. En contrepartie, l'histoire des techniques hydrauliques, donc des groupes sociaux en présence, est enrichie par l'identification de relations avec les fluctuations de « la ressource » (niveaux piézométriques, débits de l'oued, événements météorologiques).

\section{Interrogations communes sur les trajectoires «sociohydrologiques » du terrain}

À partir de ces observations croisées, les questions relatives aux trajectoires de l'espace, des eaux et des populations qui vivent avec et autour de ces "ressources" se révèlent particulièrement porteuses de convergences. La vision transitoire que nous avons l'un et l'autre des phénomènes (hydrologiques et sociaux) est à l'origine de cet intérêt commun pour ce qui a façonné le présent.

Suite à ce constat, il nous a semblé nécessaire de compléter notre vision actuelle du terrain en mobilisant les données historiques en notre possession. Cela donne lieu à une représentation de la situation antérieure à la construction du barrage (Fig. 4). Notre terrain apparaît 


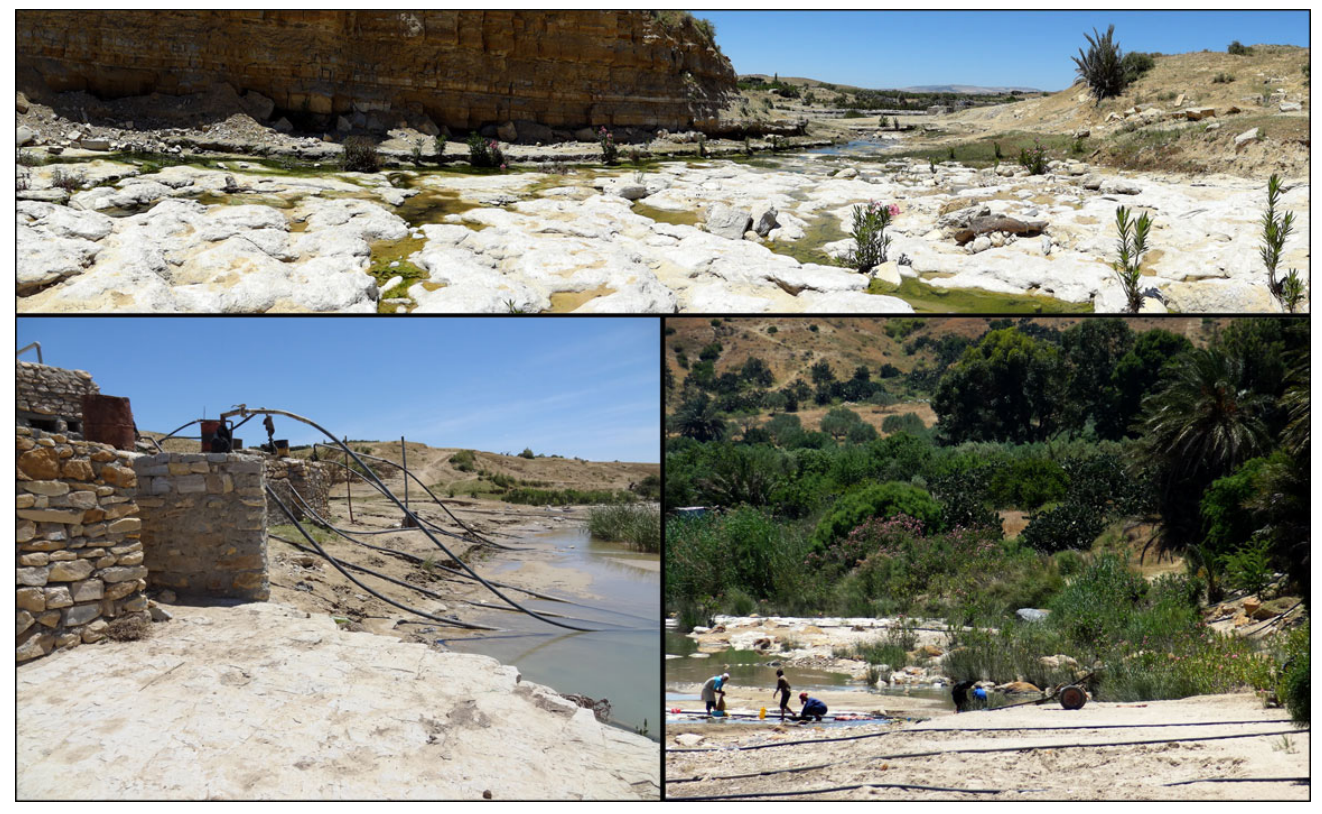

Fig. 3. La section dite « pérenne » de l'oued Merguellil, nœud de l'espace sociohydrologique.

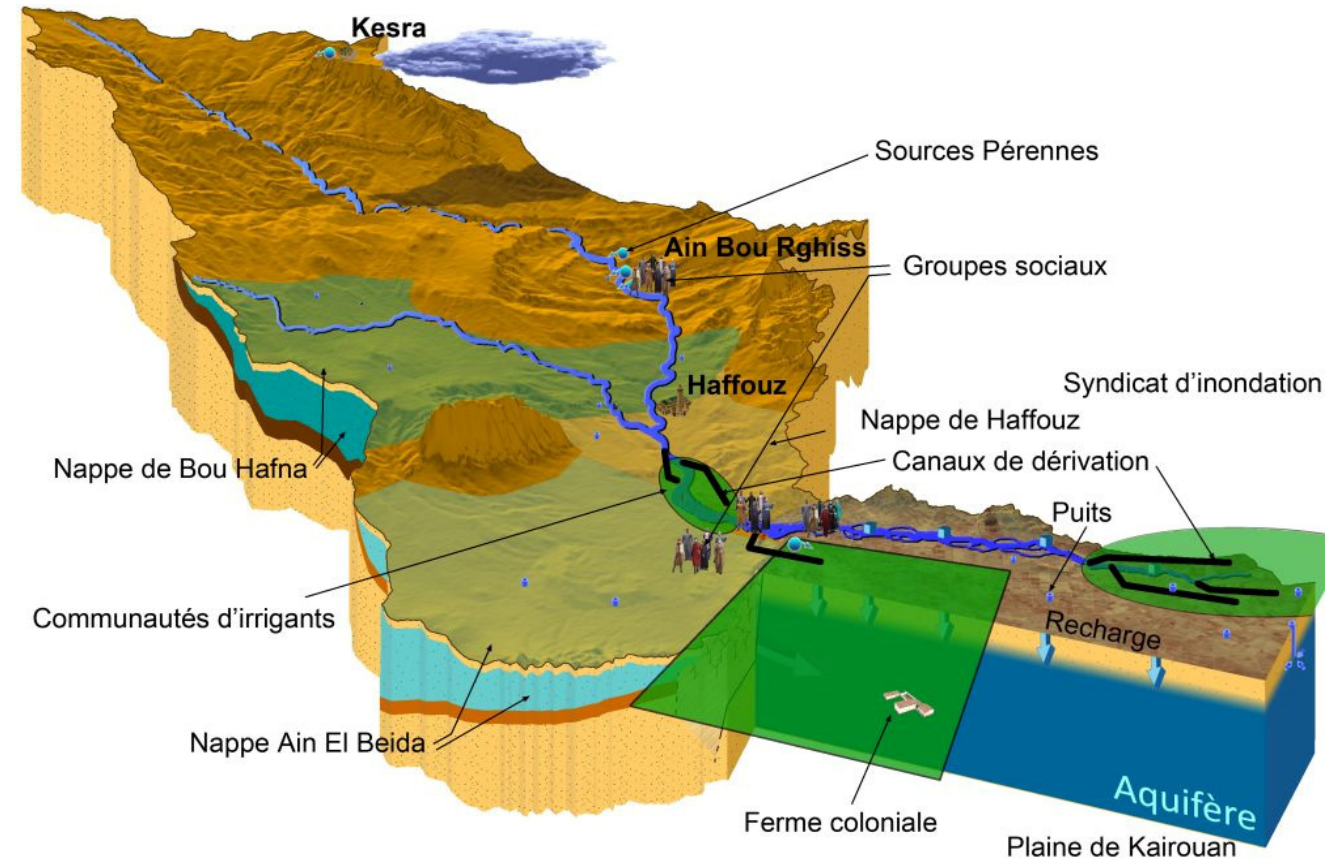

Fig. 4. Schéma « sociohydrologique » du bassin du Merguellil avant la construction du barrage.

alors complètement différent à la fois d'un point de vue « hydrologique » et « sociologique ». Les crues de l'oued Merguellil s'épandent sur la plaine favorisant l'infiltration profonde et constituent une ressource pour l'activité hydroagricole. Les grandes propriétés coloniales exploitent à la fois les eaux de crues et les eaux pérennes de l'oued (Belaïd et Riaux, 2014). Le développement des puits est encore restreint. La comparaison de la situation
« avant » et «après » l'édification du barrage entraîne des interrogations concernant la manière dont ces changements ont influencé les dynamiques hydrologiques et les organisations sociales jusqu'à produire la situation actuelle. Pour un hydrogéologue, de telles modifications impliquent de nécessaires interrogations sur l'évolution des relations entre eaux de surface et eaux souterraines : quelles évolutions des processus de recharge ont eu lieu 
et en quoi cela influe-t-il sur le constat actuel de surexploitation de la nappe de Kairouan? L'anthropologue s'intéresse aux conséquences de ces changements sur les modalités et droits d'accès à l'eau et, par conséquent, sur l'organisation sociale et politique de cet espace : comment les populations riveraines de l'oued se sont-elles adaptées à la suppression de cet accès à l'eau ?

Sur ces interrogations, chaque discipline peut avoir un apport différent, parfois complémentaire parfois supplémentaire. Ainsi l'hydrogéologie permet-elle d'expliquer à quel point les ressources mobilisables ont été réduites ou déplacées, offrant des clés de lecture en matière d'abondance et de possibilités d'exploitation ; l'anthropologie pour sa part retrace l'histoire des formes sociales, juridiques et techniques d'accès à l'eau, permettant d'émettre des hypothèses sur l'évolution des rapports sociaux et des conditions d'accès à l'eau. Le bénéfice de la collaboration interdisciplinaire est incrémental : les réponses d'une discipline invitent l'autre à approfondir ses questionnements. Nos interrogations débouchent aujourd'hui sur une problématique de recherche commune centrée sur les notions de surexploitation et d'accès à l'eau. Les résultats des travaux menés en hydrogéologie et en anthropologie s'articulent, présentant un faisceau de "preuves " pour démontrer que la baisse des niveaux de la nappe de Kairouan est plus un problème de cohérence des politiques publiques sur le long terme qu'un problème de "ressource » (Riaux et al., 2013a). Dès lors, l'enjeu majeur est celui de l'accès à l'eau, plus que celui de la préservation de la "ressource», allant à l'encontre de l'idée principale véhiculée par les administrations régionales.

\section{Conclusion}

À travers l'expérience décrite ici, l'interdisciplinarité se présente bien comme une pratique scientifique où la réflexivité doit jouer un rôle essentiel. Le processus de construction commune infléchissant fortement la démarche et les orientations de la recherche, la « conscientisation » du processus est un élément central de la méthode interdisciplinaire.

Le «terrain en commun » se prête particulièrement bien à cet exercice réflexif. Il est, en effet, simultanément support de la démarche scientifique, pratique de recherche et produit du façonnage interdisciplinaire. Nous montrons à travers son cheminement la manière dont la pratique du terrain en commun permet de générer des convergences scientifiques. Cette pratique permet d'abord de comprendre la démarche des disciplines et des chercheurs en présence, leur manière d'envisager la réalité, d'interroger, de documenter, de conceptualiser une situation. Au cours de ce processus, une définition partagée du terrain et de ses limites spatiotemporelles se dessine. Le «terrain sociohydrologique » ainsi construit se révèle propice à l'émergence d'objets et de questions de recherche interdisciplinaires. Des «notions opératoires communes » (Barrué-Pastor, 1992) émergent: la notion d'accès à l'eau vient par exemple résoudre la dichotomie entre ressources et usages. L'expérience commune du terrain nous permet de construire un filtre de compréhension partagé, qui nous amène à interroger ensemble la notion de surexploitation véhiculée par les administrations tunisiennes à la lumière de cette notion d'accès à l'eau. À travers ces «nœuds sociohydrologiques » du terrain et de l'analyse apparaissent des objets, lieux et moments pour lesquels l'association de l'hydrogéologie et de l'anthropologie fait sens. En organisant notre collaboration autour de ces «nœuds ", nous ne forçons pas les convergences ; recherches disciplinaires et interdisciplinaires s'articulent et s'alimentent réciproquement. Chacun de nous peut alors être « indiscipliné » tout en conservant un ancrage fort dans sa discipline (Barrué-Pastor, 1992 ; Riaux et al., 2013b).

À travers cette perspective réflexive, on constate qu'autour et au cours du terrain différents problèmes inhérents à l'interdisciplinarité ont été soulevés et résolus. C'est le cas de la définition des échelles d'espace et de temps, de l'élaboration d'un vocabulaire partagé, $d^{\prime}$ 'une représentation commune de la situation, du choix $d^{\prime}$ angles d'analyse qui fassent sens pour nos deux disciplines, etc. La manière dont ces écueils potentiels sont apparus et les réponses que nous y avons apportées reposent sur la spécificité du dispositif de recherche élaboré ensemble. Entrent en jeu, bien sûr, nos individualités, nos trajectoires personnelles de recherche et une bonne entente : ingrédients nécessaires au bon déroulement d'une recherche interdisciplinaire. Toutefois, d'autres caractéristiques de l'interdisciplinarité que nous construisons infléchissent très largement l'orientation de notre démarche et ses résultats par rapport à $\mathrm{d}$ 'autres façons de penser et de pratiquer la recherche interdisciplinaire.

L'interdisciplinarité que nous mettons en œuvre est une interdisciplinarité « endogène » et « fondamentale » (Jollivet et Legay, 2005). Elle est endogène, car même si le projet qui a donné lieu à notre collaboration nous précède, nous ne sommes pas sollicités dans le cadre d'un programme de recherche déjà défini. Notre présence sur un même terrain résulte d'une volonté de franchissement des frontières déjà ancienne à l'IRD (Couty, 1993) et qui fait aujourd'hui écho à une évolution plus générale de la posture des sciences de l'environnement, bien illustrée par Harris et Lyon (2013). En revanche, la manière $\mathrm{d}$ 'associer les sciences de l'environnement et les sciences sociales n'est ni imposée ni même pensée a priori, les résultats attendus non plus. L'une des forces de notre collaboration réside probablement dans le fait que l'interdisciplinarité entre anthropologie et hydrogéologie ne 
repose pas sur des précédents déjà établis. Il y a dès lors dans cette forme d'interdisciplinarité un aspect exploratoire qui relève de la recherche fondamentale, à la fois dans la démarche à mettre en œuvre et dans les résultats auxquels elle aboutit.

Ce contexte de travail se révèle très différent $\mathrm{d}$ 'autres formes d'interdisciplinarité focalisées sur la résolution d'une question de recherche préalablement définie, souvent dictées par des injonctions émanant des politiques de recherche et des bailleurs de fonds (cf. p. ex. Bruce et al., 2004). Alors que nous progressons de manière inductive et itérative, à travers un «frottement » entre disciplines par l'intermédiaire du terrain, les programmes interdisciplinaires impliquent souvent un processus de discussion " en salle », préalable à la recherche elle-même, où le dialogue entre disciplines est orienté par les objectifs à atteindre (Jeffrey, 2003). Mais, le problème ne réside pas dans la définition "exogène » et «finalisée » de la question scientifique, qui serait même l'un des moteurs de l'interdisciplinarité (p. ex. Jollivet et Legay, 2005 ; Barruet-Pastor, 1992). Le problème réside plutôt dans la place que l'on accorde à l'expérience directe et partagée du terrain. Comme l'ont montré Mathieu et al. (1997), c'est en se confrontant ensemble au concret, sur le terrain, qu'une redéfinition partagée de la question sociétale, donc de la manière d'y répondre, est rendue possible. D'ailleurs, dans notre cas, si le regard sociohydrologique part d'une posture de recherche «fondamentale», son ancrage sur le terrain suscite la construction d'interrogations qui relaient celles des acteurs avec lesquels nous interagissons. Dès lors, les questions qui font sens pour nous font écho à leurs préoccupations, rejoignant ainsi des questions de société. Une interdisciplinarité plus "finalisée » semble dès lors se profiler dans la continuité de notre recherche «fondamentale».

\section{Remerciements}

Nous remercions Z. Jenhaoui dont la médiation linguistique a rendu possible le travail de terrain auprès des acteurs de l'eau, ainsi que l'ensemble des membres de l'équipe G-eau/Inat qui donne aussi vie à l'environnement interdisciplinaire dans lequel nous évoluons.

\section{Références}

Aubriot, O., Riaux, J. (Eds), 2013. Savoirs sur l'eau, techniques et pouvoirs, Autrepart, 65.

Barrué-Pastor, M., 1992. L'interdisciplinarité en pratiques, in Jollivet, M. (Ed.), Sciences de la nature, sciences de la société : les passeurs de frontières, Paris, CNRS, 457-475.
Belaïd, H., Riaux, J., 2014. Appropriation et gestion des eaux en Tunisie à l'époque coloniale : une histoire de dépossession(s) ? Cas du Kairounnais, Rawafid (Revue de l'Institut d'histoire du mouvement national) [à paraître].

Bruce, A., Lyall, C., Tait, J., Williams, R., 2004. Interdisciplinary integration in Europe: The case of the Fifth Framework Programme, Futures, 36, 4, 457-470.

Couty, P., 1993. Le cercle des sciences: réflexions sur la recherche multidisciplinaire, Cahier des Sciences Humaines, 29, 1, 231-237.

Fabre, E., Cantelaube, J., 2011. Le loup, la métairie et la forge : dialogue entre un historien et un écologue, Natures Sciences Sociétés, 19, 272-276.

Favreau, G., Leduc, C., Marlin, C., 2000. Reliability of geochemical and hydrodynamic sampling in a semi-arid water table, Journal of African Earth Sciences, 31, 3-4, 669-678.

Harris, F., Lyon, F., 2013. Transdisciplinary environmental research: Building trust across professional cultures, Environmental Science \& Policy, 31, 109-119.

Jeffrey, P., 2003. Smoothing the waters: Observations on the process of cross-disciplinary research collaboration, Social Studies of Science, 33, 4, 539-562.

Jollivet, M. (Ed.), 1992. Sciences de la nature, sciences de la société : les passeurs de frontières, Paris, CNRS.

Jollivet, M., Legay, J.-M., 2005. Canevas pour une réflexion sur une interdisciplinarité entre sciences de la nature et sciences sociales, Natures Sciences Sociétés, 13, 184-188.

Latour, B., 1993. Le «pédofil » de Boa Vista : montage photophilosophique, in Latour, B., Petites leçons de sociologie des sciences, Paris, La découverte, 171-225.

Le Goulven, P., Leduc, C., Bachta, M.-S., Poussin, J.-C., 2009. Sharing scarce resources in a Mediterranean River Basin: Wadi Merguellil in Central Tunisia, in Molle, F., Wester, P. (Eds), River Basin Trajectories: Societies, Environments and Development, Wallingford, CABI, 147-170.

Leduc, C., Ben Ammar S., Favreau, G., Béji, R., Virrion, R., Lacombe, G., Tarhouni, J., Aouadi, C., Zenati Chelli, B., Jebnoun, N., Oi, M., Michelot, J.-L., Zaoauri, K., 2007. Impacts of hydrological changes in the Mediterranean zone: Environmental modifications and rural development in the Merguellil catchment, Central Tunisia, Hydrological Sciences Journal, 52, 6, 1162-1178.

Marzouk, Y., 1989. Sociétés rurales et techniques hydrauliques en Afrique, Études Rurales, 115-116, 9-36.

Massuel, S., Perrin, J., Wajid, M., Mascre, C., Dewandel, B., 2008. A simple, low-cost method to monitor duration of ground water pumping, Ground Water, 47, 1, 141-145.

Massuel, S., Cappelaere, B., Favreau, G., Leduc, C., Lebel, T., Vischel, T., 2011. Integrated surface water-groundwater modelling in the context of increasing water reserves of a regional Sahelian aquifer, Hydrological Sciences Journal, 56, 7, 1242-1264.

Mathieu, N., Rivault, C., Blanc, N., Cloarec, A., 1997. Le dialogue interdisciplinaire mis à l'épreuve: réflexions à partir d'une recherche sur les blattes urbaines, Natures Sciences Sociétés, 5, 1, 18-30.

Olivier de Sardan, J.-P., 1996. La violence faite aux données, Enquête, 3, 31-59. 
Pillon, P., 2008. Sciences sociales en situations asymétriques, Natures Sciences Sociétés, 16, 364-367.

Quensière, J., 1997. Autour des blattes urbaines: quelques réflexions sur l'interdisciplinarité, Natures Sciences Sociétés, 5, 34-37.

Riaux, J., 2006. Règles de l'État-règles de la communauté: une gouvernance locale de l'eau (Haut Atlas, Pyrénées). Thèse de doctorat en anthropologie sociale, EHESS, Paris.

Riaux, J., 2013. Engager la construction d'un regard sociohydrologique : des archives catalyseurs de l'interdisciplinarité, Natures Sciences Sociétés, 21, 15-23.

Riaux, J., Massuel, S., Burte, J., 2013a. Reformuler les interrogations sur la surexploitation des eaux souterraines : renversement des regards sur la nappe de Kairouan, in Kuper, M. (Ed.), Actes du Séminaire Groundwater-Arena "La gouvernance des eaux souterraines au Maghreb », Biskra, Algérie, 3-7 déc. (online : http:/ / www.groundwater-arena.net/content/download $/ 4167 / 31185 /$ version/1/file/Actes+Biskra+Arena. pdf).

Riaux, J., Massuel, S., Barreteau, O., 2013b. Expériences interdisciplinaires: quels retours sur les disciplines? Table ronde avec P. Cornu et J.-P. Billaud, Colloque "Interdisciplinarités entre natures et sociétés ", Association NSS-Dialogue/revue NSS, Cerisy-la-Salle, Centre culturel international, 30 sept. -5 oct.

Reçu le 12 mai 2013. Accepté le 3 juillet 2014. 